Aus dem städtischen Krankenhaus zu Konstanz (dirig. Arzt: Med.-Rat Dr. O. Kappeler).

\title{
Die Ruptur des inkarzerierten Darmes bei der Taxis.
}

\author{
Von Dr. 0. Kappeler.
}

(Mit Tafel III.)

Die Berstungsruptur des gesunden, nicht ulzerierten und noch nicht der Gangrän verfallenen Darmes durch forcierte oder ungeschickte Taxisversuche wird von manchen Chirurgen und auch von erfahrenen Herniologen teils geleugnet, teils als sehr unwahrscheinlich hingestellt. So sagt $\mathrm{z} . \mathrm{B}$. L i n $\mathrm{h}$ a $\mathrm{r} \mathrm{t}$ in seinen Vorlesungen über Unterleibshernien: „Daß ein Bersten des Darms beim Zurückbringen o p e r i e r t e r Hernien möglich ist, habe ich selbst schon einige Male gesehen, daß aber ein gesunder Darm durch die Haut hindurch bei der Taxis zum Bersten kommt, ist ungemein selten, er müßte bereits in einem gewissen Ulzerationsstadium sich befinden oder durch fibrinöse Exsudationen brüchiger geworden sein. Ist eine große Menge Serum vorhanden, so ist das Bersten vollends unmöglich und nur, wenn man den Darm zusammendrückt, kann es geschehen, daß eine weit hinunter reichende Darmschlinge einer Ruptur ausgesetzt sein kann. Von manchen Ärzten wird eine solche Kraft angewendet, daß sie sich mit den Knien gegen den auf der Erde liegenden Patienten stemmen, denn man findet manchmal die Spuren der Nägel des Chirurgen, Hautabschürfungen, Sugillationen, und doch tritt keine Berstung ein; dagegen kann eine forcierte Taxis, besonders wenn sie ungeschickt ausgeführt wird, Quetschung des Darms hervorrufen."

Nach M a y d 1 kann man die Möglichkeit einer Darmruptur durch Taxis nur dann zugeben, wenn zuerst der Bruchsack einreißt und dann erst durch weitere Manipulationen auch die durch den Riß vorgedrängten Därme gewissermaßen zerwühlt würden. 
Heute verfügen wir über ganz sichere Beobachtungen von Darmruptur bei gesundem oder durch die Inkarzeration sehr wenig geschädigtem Darm. Sie scheinen allerdings noch selten zu sein ich bin in der mir zugänglichen Literatur nur auf 4 Fälle gestoßen - einen weitern, noch unveröffentlichten Fall verdanke ich meinem geschätzten Kollegen Dr. C. B r u n n e r, Chefarzt in Münsterlingen -, allein sie sind trotzdem vielleicht nicht so selten, als es scheinen möchte, denn die Fälle der allgemeinen Praxis, wo dic Diagnose nicht gestellt, ein weiterer Eingriff unterlassen und eine Sektion nicht gemacht wurde, sind wohl von vornherein von de1 Veröffentlichung ausgeschlossen. Bei der angeblichen Seltenheit des folgenschweren Ereignisses, ist es doch auffallend, daß mir allein schon 3 Taxisperforationen begegnet sind, und 2 davon in kurzem Intervall. Als nebensächlich darf jedenfalls die Berstung des Darms bei der Beurteilung der Taxis und ihren Folgen nicht hingestellt werden, und ihre Möglichkeit ist schon an und für sich dazu angetan, den von verschiedenen Seiten erhobenen Ruf: ,Weg mit der Taxis!" aufs kräftigste zu unterstützen.

\section{a. Eigene Beobachtungen:}

I. Am 9. III. I908 wurde die 4Ijährige, verheiratete Frau M. M. in das hiesige städtische Krankenhaus gebracht. Nach ihren Mitteilungen war sie früher gesund. Vor 3 Jahren bekam sie in der rechten Leistengegend eine walnußgroße Geschwulst, die allmählich größer wurde und schlịßßlich $\mathrm{Hühnereigröße} \mathrm{erreichte,} \mathrm{von} \mathrm{der} \mathrm{Pa}$ tientin leicht in die Bauchhöhle zurückgeschoben werden konnte und weiter keine Schmerzen oder anderweitige Beschwerden verursachte. Von Zeit zu Zeit kam sie wieder zum Vorschein, konnte aber ohne Schwierigkeit jeweils wieder reponiert werden. Am 8. III. früh hatte sie noch Stuhl. Nachmittags 2 Uhr trat der Bruch wieder aus unter heftigen Schmerzen im Leib und im Bruch, 2 Stunden später stellte sich heftiges Erbrechen ein, worauf der Bruch noch größer wurde. Die Schmerzen dauerten an und wurden gegen $A$ bend so heftig, da $B$ Patientin den Arzt kommen ließ, der abends 1/2 7 Uhr eintraf und etwa $1_{1}$ Stunde lang Repositionsversuche machte, die so schmerzhaft waren, $\mathrm{da} B$ Patientin laut aufschrie. Der Bruch wurde etwas kleiner, verschwand aber nicht ganz, die Schmerzen aber nahmen an Heftigkeit $\mathrm{zu}$, es traten wieder Brechreiz und wiederholtes Erbrechen auf, das die ganze Nacht mit kurzen Unterbrechungen anhielt. Auch wurde sie oft schwindelig und ohnmächtig vor Schmerz und konnte vor Schwäche kaum mehr im Bett aufsitzen. 
Als sic am 9. III. vormittags 9 Uhr ins Spital kam, sah sie sehr angegriffen, aber nicht eigentlich kollabiert aus. Puls 76, Temp. 37,5. Unterleib kaum aufgetrieben, in den unteren Partien druckempfindlich, Bauchdecken nicht gespannt. In der rechten Schenkelbeuge findet sich unter dem Lig. Pouparti eine etwa hühnereigroße, weiche, teigige, schon auf leise Berührung sehr schmerzhafte Geschwulst. Die über der Geschwulst bewegliche Haut ist stellenweise schmutzig. rot und grün verfärbt. In beiden Hypochondrien helltympanitischer Schall. Leberdämpfung vorhanden. Nach sorgfältiger Reinigung des Operationsfeldes wird ohne weiteren Verzug in guter Äthernarkose ein $12 \mathrm{~cm}$ langer, vertikaler Schnitt über die Hernic geführt und nach Durchtrennung des blutig suffundierten Zellgewebes und der Fascia propria der unversehrte Bruchsack bloßgelegt. Bei Eröffnung des Bruchsacks fließen nur einige Tropfen blutigen Serums ab, und nun kommt die Kuppe einer tief blauschwarzen Dünndarmschlinge zum Vorschein, die mit schwarzen, lockern Blutgerinnseln bedeckt ist. Nach sorgfältigem Wegwischen der Blutgerinnsel sieht man auf dem höchsten Punkt der Kuppe ein erbsengroßes Loch in der Darmwandung ohne Schleimhautprolaps, durch das die Sonde widerstandslos in das Lumen des Darmes eindringt. Bei Druck auf den Darm fließt ganz wenig blutige Flüssigkeit heraus. Da die Abschnürung der inkarzerierten Darmschlinge eine außerordentlich enge ist, so da $B$ nicht einmal das Kaufmannsche Herniotom in den Bruchring eingeführt werden kann, wird die Bruchpforte von außen nach innen durchtrennt und der Darm nach provisorischem Abschluß der Perforationsöffnung vorsichtig vorgezogen. Er zeigt eine gelbgraue, breite, glanzlose Schnürfurche, und der zuführende Schenkel ist bis auf etwa $10 \mathrm{~cm}$ vom Bruchring auf wärts gebläht und injiziert. Aus der Peritonealhöhle höhle kommt kein Tropfen Flüssigkeit.

Da die Darmschlinge offenbar der Gangrän verfallen, entschließe ich mich kurzer Hand zur Darmresektion im Gesunden; es werden ca. $20 \mathrm{~cm}$ des zuführenden und $8 \mathrm{~cm}$ des abführenden Schenkels der eingeklemmten Darmschlinge nach Lostrennung und Unterbindung des Mesenteriums rezesiert und die beiden Lumina end to end, nachdem ihr Umfangsunterschied durch schiefe Durchtrennung des abführenden Schenkels ausgeglichen war, durch fortlaufende Serosamuscularis-Naht und fortlaufende Mucosanaht aneinander genäht, Faltung und Vernähung der abgetrennten Mesenterialplatte. Dann Isolierung des Bruchsacks, Abbindung und Naht der Bruchpforte.

Die Heilung erfolgt ohne $Z$ wischenfälle, nachdem schon am II. III. auf Ölklysma der erste Stuhl abgegangen war.

Der Befundbericht über die mikroskopische Untersuchung der resezierten Darmschlinge von Herrn Dr. M ü 1 l e r, Assistent am pathologischen Institut in Tübingen, lautet wie folgt: An der Schnürfurche beginnt die Mucosa und Submucosa eine undeutliche Kernfärbung zu 
erhalten. Nach der Perforationsstelle zu nimmt diese Erscheinung an Stärke und Ausdehnung allmählich zu. Etwa in der Mitte zwischen Schnürfurche und Perforation wird auch die Muscularis nekrotisch. In unmittelbarer Umgebung der Perforationsöffnung ist die ganze Wand nur noch eine nekrotische, da und dort mit Leukocyten durchsetzte Masse. Außerdem ist in ganzer Ausdehnung des eingeklemmten Stückes die Wand hämorrhagisch infiltriert, ebenfalls von außen nach innen zunehmend, die Gefäße, soweit erhalten, dicht gefüllt mit Blut. Auch die Oberfläche ist mit Blutgerinnseln bedeckt.

2. Am 4. X. Igo8 wurde ich telegraphisch aufs Land gerufen mit der kurzen Angabe, es möchte sich um eine Scheinreduktion bei einer inkarzerierten Schenkelhernie handeln.

An Ort und Stelle angelangt, fand ich eine 48 jährige Frau, die seit einem halben Jahre an einer Schenkelhernie litt, die durch ein Bruchband bis jetzt so gut zurückgehalten wurde, daB sie nie austrat und überhaupt keine Beschwerden machte.

Die Frau klagte über sehr heftige, fast unerträgliche Schmerzen im ganzen Bauch. Aussehen matt und angegriffen, tiefliegende Augen. Puls I I6, klein. Abdomen wenig gespannt, stark meteoristisch aufgetrieben, und durch die dünnen Bauchdecken zeichnen sich deutlich geblähte Darmschlingen ab. Leiser Druck schon sehr empfindlich. Leberdämpfung klein, im linken Hypochondrium matter, bei Seitenlage sich aufhellender Schall. Der behandelnde Arzt machte folgende Angaben:

Am 3. X. abends $1 / 28$ Uhr wurde ich wegen ziemlich stürmischen Inkarzerationserscheinungen seitens eines taubeneigroßen linksseitigen Schenkelbruchs zu der Frau gerufen: Der Bruch war vor $21 \frac{1}{2}$ Stunden ausgetreten. Ich versuchte die Taxis, und nach 7 Minuten langem, vorsichtigem Manipulieren gelang es mir, die Hernie zurückzubringen. Die Frau äußerte im ersten Moment ein Gefühl der Erleichterung zu haben. Doch kurze Zeit nachher klagte sie wiederum über rasende Schmerzen im ganzen Bauch bis zum Anus herunter. In der Nacht mehrmals Erbrechen. Trotz Anwendung von Opium heftige Schmerzen bis zum andern Mittag und vormittags, am 4. X. wiederholtes Erbrechen. Diagnose: Reposition en bloc.

Ich äußerte mich dahin, es möchte sich nach den vorliegenden Symptomen einer perakuten, allgemeinen Peritonitis wohl eher um eine durch die Taxis bewirkte Darmperforation handeln und schlug, da wegen der schlechten Zugverbindung ein baldiger Transport ins Krankenhaus unmöglich war, die sofortige Operation in loco vor, trotz der sehr ungünstigen äußern Verhältnisse und der mangelhaften Assistenz. Es waren seit der Taxis 20 Stunden verstrichen.

Nach den üblichen Vorbereitungen wird in Äthernarkose der Schenkelring freigelegt, aus dem ein leerer, schlaffer, etwa $5 \mathrm{~cm}$ langer Bruchsack heraushängt. Nach Eröffnung desselben fließt 
etwas gelbgrüner, stinkender Eiter ab. Die Bruchpforte ist für den Zeigefinger durchgängig. Nun Verlängerung des Schnittes nach oben durch die Bauchdecken und Eröffnung der Bauchhöhle, worauf fäkalriechender, mißfarbiger Eiter in ziemlicher Menge abfließt. Die sich vordrängenden Dünndarmschlingen sind gebläht, stark injiziert, mit Fibringerinnseln bedeckt und locker durch solche miteinander verklebt. Beim vorsichtigen Vorziehen einer der unteren der Bauchwand anliegenden Schlingen, zeigt sich auf deren Konvexität ein bohnengroßes Loch mit gerissenen Rändern und Schleimhautvorfall, aus dem dünner, gelber Kot im Strahl herausfließt. Die Umgebung der Perforationsstelle zeigt sonst keine weiteren Veränderungen, sie ist nicht stärker injiziert als der übrige Darm, und nirgends findet sich beim Absuchen dieser Schlinge auch nur eine Andeutung von Schnürfurche.

Abklemmung dieser Darmschlinge auf beiden Seiten und quere Übernähung der Perforationsöffnung mit doppelter, fortlaufender Zwirnnaht. Dann bestmögliche, trockene Reinigung des Beckens und der linken Bauchseite, die hauptsächlich mit dem fäkulenten Eiter überschwemmt sind, während nach rechts hin durch Verklebung der Darmschlingen eine Art Schutzwall gegen die Propagation des kotigen Eiters zustande gekommen war. Einführung einer großen Vioformmèche in die gereinigte Beckenhöhle und teilweise dreifache Naht der Bauchwunde.

Etwa 4 Wochen später erhielt ich vom behandelnden Arzt einen kurzen Bericht, der folgendermaßen lautet: Die Patientin hatte nach der Operation keine erhöhten Temperaturen, der Puls war noch 3 Tage beschleunigt (100-120) und klein. Am 4. Tage wurde die Vioform mèche entfernt. Seither ging die Heilung gut, wenn auch nicht per primam, vonstatten. Am 5. Tag erfolgte Stuhlklysma, und hernach alle andern Tage von selbst. Die Frau ist seit 3 Tagen außer Bett und es geht ihr ausgezeichnet.

3. Ein 48jähriger sehr energischer und kräftiger Fabrikant litt schon seit Jahren an einer linksseitigen Inguinalhernie, die trotz Bruchband von Zeit zu Zeit austrat. Patient reponierte die Hernie immer selbst und die Reposition gelang bis jetzt ohne Schwierigkeit. Eben im Begriff, ein wichtiges Geschäft abzuschließen und zu diesem Zwecke eine längere Reise anzutreten, trat der Bruch wieder aus. Patient machte sich sofort wieder an die Reposition, die diesmal nicht glücken wollte. Er drückte immer stärker und stärker die etwa ganseigroße Geschwulst zusammen, als plötzlich unter einer Art Krachen der Bruch verschwand. Er bekam sofort heftige Bauchschmerzen und mußte sich zu Bett legen.

Drei Tage später sah ich den Kranken konsultativ, mit fadenförmigem Puls von I60, fliegender Respiration, Schwarzbrechen und allen Symptomen einer allgemeinen Peritonitis. Von einer Operation konnte 
keine Rede mehr scin. Im Verlaufe des gleichen Tages Exitus. Die Vermutung einer Darmruptur infolge forcierter Taxis wurde durch die Obduktion bestätigt. Es fand sich in einer unteren Iliumschlinge ein $2 \mathrm{~cm}$ langer perforierender Riß der Darmwand.

b. Der folgende Fall wurde im Kantonspital Münsterlingen beobachtet und von Dr. C. Brunner operiert:

4. W. Chr., 57jähriger Landwirt, wurde am 28. VI. 1908 abends $61 / 2$ Uhr dem Kantonsspital Münsterlingen zugeführt mit folgendem Zeugnis des behandelnden Arztes: Der Mann leidet schon seit Jahren an einer rechtsseitigen Inguinalhernie, zu deren Reposition er sich jeweils eines Stockes bediente, gegen den er sich mit seinem Körpergewicht anstemmte. Als er diese Manipulation am 27. VI. abends ausführte, hörte er plötzlich ein eigentümliches Kollern und gleichzeitig verspürte er heftige Schmerzen im ganzen Abdomen, auch mußte er mehrmals erbrechen. Als diese Erscheinungen nicht nachlieBen und auch Stuhl und Winde ausblieben, lieB mich Patient heute nachmittag (28. VI.) rufen.

Status bei der Aufnahme: Großer, magerer Mann, keine Facies peritonitica. Temperatur 38,1. Puls klein, weich, anfangs 80, später 120. Vorwiegend kostale Atmung, oberflächlich 56. Leberdiimpfung verkleinert. Singultus. Sofortige Kochsalzinfusion mit Adrenalin intermuskulär. Abdomen wenig gespannt, auf Druck schmerzhaft und in der Blinddarmgegend Dämpfung. Hernie reponiert. Stuhl gestern morgen. Winde gestern abend. Diagnose: Peritonitis nach Darmperforation. Operation ca. 24 Stunden nach der forcierten ' $\Gamma$ axis in Äthertropfnarkose. Schnitt über der Dämpfung in der Ileocöcalgegend. Bei Eröffnung des Peritoneums strömt ein Strahl Eiter und Darminhalt hervor. In der lleocöcalgegend findet sich ein Paket durch Fibringerinnsel verklebter Darmschlingen, bei deren Trennung ein ca. I $\mathrm{cm}$ im Durchmesser messendes Loch im Ileum an der Ansatzstelle des Mesenteriums zum Vorschein kommt. Provisorische Naht des Lochs und Absuchen des ganzen Darmtraktus. Alle Därme stark mit Fibrin belegt. Peritoneum parietale stark entzündet. Ausspülung des Abdomens mit ca. I7 Liter Kochsalzlösung. Einnähen der Perforation in die Wunde. Links im Unterbauch Kontrainzision und Einführen eines starken Drains. Puls nach der Operation kaum fühlbar. Kochsalzinfusion intravenös.

Die Schmerzen ließen nach der Operation nach, und aus der Fistel floß massenhaft dünner, schwarzer Stuhl. Exitus Io Stunden nach der Operation.

Die Sektion ergab eine Peritonitis diffusa.

c. In der mir zugänglichen Literatur fand ich folgende Fälle von Darmberstung durch Taxis: 
5. Hut chin on und $\mathrm{Jacks}$ on beschreiben einen eingeklemmten Schenkelbruch bei einem 57jährigen Manne, wo die Taxis allein vorgenommen war und der Exitus 26 Stunden nach derselben erfolgte. Bei Autopsie ergab sich eine Darmperforation an der eingeklemmt gewesenen Darmpartie.

6. R. F r a n k beschreibt in der Wiener med. Wochenschrift $\mathrm{I} 892$ einen Fall von Perforationsperitonitis durch Ruptur des Darmes bei der Taxis: Eine 53jährige Kranke litt schon seit längeren Jahren an einem linksseitigen Schenkelbruch, der schon wiederholt sich eingeklemmt hatte, aber jedesmal durch einen Arzt wieder reponiert werden konnte. Am 8. VII. 1892 abends 6 Uhr Wiedereinklemmung. Reposition durch den Arzt nach kaum einer Stunde, ohne besondere Gewalt, rasch und unter deutlichem Gurren. Schon bei der Reposition heftiger Schmerz, der rasch zunahm, und Erbrechen. Eine Stunde nach der Reposition shokierte Gesichtszüge, beschleunigter kräftiger Puls, Meteorismus mit Bauchdeckenspannung, Druckempfindlichkeit und nachweisbares Exsudat. Rasche Verschlimmerung, Zunahme des Kollapses, Singultus und fäkulentes Erbrechen. 4 Stunden nach der Taxis Herniolaparotomie: Bruchsack leer, entleert stark trübe Flüssigkeit. Dünndarmschlingen geschwellt, injiziert und mit graugelbem, fleckigem Exsudat belegt. An einer dunkler gefärbten, stärker geschwellten Schlinge eine $4 \mathrm{~mm}$ lange, schlitzförmige, quergestellte Lücke, aus der Gasblasen aufstiegen und flüssiger Darminhalt im Strahle ausrann. Naht des Lochs. Jodoformgazetamponade. Heilung.

7. Einen weiteren Fall teilt $\mathrm{Kr}$ a $\mathrm{ft}$ aus der chirurg. Klinik zu Rostock (Professor Garrè) mit: Patient hat einen seit Jahren bestehenden Bruch, der immer reponibel gewesen ist. Plötzlich klemmte er sich eines Tages ein. Patient selbst und seine Angehörigen machten intensivste, aber erfolglose Taxisversuche. Der Leib schwoll an und wurde druckempfindlich. Der darauf zugezogene Arzt stellt die Diagnose auf inkarzerierte Cruralhernie und riet sofort, ohne erst Taxisversuche zu unternehmen, dringend zur Operation. Gegen das ärztliche Verbot wurde nochmals ohne Erfolg die Reposition versucht. Dann Herniolaparotomie (Temperatur vor der Operation 37,8, Puls 104): Bruchsack leer, mit Fibringerinnseln ausgekleidet. Aus dem Peritoneum fließt reichlich mißfarbiger Eiter aus. Darmschlingen gebläht und miteinander verklebt durch festaufsitzende fibrinös-eitrige Auflagerun. gen. Bei der Lösung der verklebten Dünndarmschlingen findet sich an einer Stelle ein etwa 2o-pfennigstückgroßes Loch, das provisorisch durch eine angelagerte Schlinge verklebt war, aus der dünner Kot ausflieBt.

8. Schnitzler (Wiener med. Wochenschr. I 899 und Centralblatt für Chirurgie 1900) erzählt von einer 56jährigen Frau, die seit 3 Wochen an einem rechtsseitigen Schenkelbruch leidet. Eines Tages 
wird der Bruch irreponibel und schmerzhaft. Am Abend gelingt es der Kranken, den Bruch zurückzubringen, aber die Schmerzen lassen nicht nach. Abends Erbrechen. Am nächsten Morgen Diagnose: Peritonitis, wahrscheinlich durch Perforation. Nachweis eines Exsudats. Operation 18 Stunden nach der Taxis. Am Bruchsack frische Auflagerungen, die ganze Peritonealhöhle im Zustand fibrinös-eitriger Entzuindung. Ausgangspunkt derselben ein bohnengroßes Loch im mittlern Ileum. Umgebung desselben zeigt keine Spuren, da $B$ der Darm früher schon pathologisch verändert war. Anfrischung des Defektes. Naht. Heilung.

Schon der Umstand, da $B$ unter den acht Beobachtungen über Ruptur des Darmes durch die Taxis eingeklemmter Brüche sich 4 Fälle von Selbsttaxis finden, spricht wohl dafür, daß die Taxisversuche in unzweckmäßiger und aus Angst, dem Chirurgen in die Hände zu fallen, in forcierter Weise ausgeführt wurden. Nur bei den Beobachtungen 2 und 6 ist ausdrücklich erwähnt, daß auf Taxis nur kurze Zeit verwendet und da $B$ mit Schonung vorgegangen wurde.

Der Mechanismus dieser Art von Darmruptur liegt offen zutage:

Nach den Angaben des Befundes, die von hühnerei- bis gänseeigroßen, derben, prallen Bruchgeschwülsten reden, findet sich im Bruchsack ohne schützendes Netz eine mit Gas und Flüssigkeit gefüllte Darmschlinge, die nach beiden Seiten hin abgeklemmt und festgehalten ist. Wird nun durch Taxisversuche auf den Darm und das in ihm befindliche Gasflüssigkeitsgemenge ein starker Druck ausgeübt, so platzt der Darm, wo der geringste äuBere Widerstand vorhanden ist, d. h. gewöhnlich an der Kuppe, die durch venöse Stauung am meisten gelitten hat und wie dies bei meinen beiden Beobachtungen de facto der Fall ist. Besonders verhängnisvoll wird der Druck sein, wenn, wie im Falle S c h n i t z l e r und bei meiner ersten Beobachtung, der Bruchsack leer ist und der innere Druck im Darm keinem Gegendruck begegnet.

Was nun den weiteren Verlauf der erfolgten Berstung betrifft, so hängt dieser lediglich davon $a b$, ob die geborstene Schlinge ihren Inhalt nur in den Bruchsack entleert, was nur möglich ist, wenn er auch nach der Berstung von der freien Bauchhöhle abgeschlossen bleibt, wie in meinem ersten Falle, oder ob durch Hineinfließen des Bruchsackinhalts oder durch Zurückschlüpfen der 
rupturierten Darmpartie die Bauchhöhle mit infektiösem Inhalt überschwemmt wird.

Im ersten Fall spielt sich die ganze Katastrophe nur im Bruchsack ab. Ist, wie bei unserem Kranken, der Darm leer und die Abschnürung trotzdem eine sehr enge, so dauern die Inkarzerationserscheinungen in ungeschwächter Weise fort, aber die Peritonitis läßt auf sich warten. Wird dann der Bruchsack beizeiten eröffnet, so braucht das ganze therapeutische Verfahren nur in der Naht der Darmlücke und einer sorgfältigen Reinigung des Darmes und des Bruchsackes oder besser in einer Exstirpation des mehr weniger entzündeten Bruchsackes und in der Reposition der eingeklemmten Schlinge zu bestehen. Ist der Darm dagegen schon gangränös oder gangränverdächtig, so bietet, wie obiger Fall zeigt, bei der Intaktheit der großen Peritonealhöhle die Resektion des gangränösen Darmes die besten Aussichten.

Anders im zweiten Fall.

Hier setzt sofort eine sich rasch auf das ganze Peritoneum oder auf einen großen Teil desselben sich ausbreitende Entzündung ein, die, wenn ein Eingriff unterlassen wird, fast unfehlbar ad exitum führt und auch bei rechtzeitigem Eingreifen viele Gefahren in sich birgt.

Eine Differentialdiagnose dieser beiden Zustände wird wohl manchmal zu machen sein.

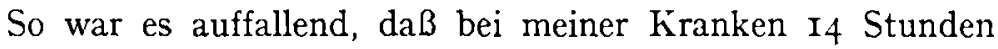
nach der Taxis, d. h. nach erfolgter Ruptur, der Puls verhältnismäßig langsam, die Temperatur kaum erhöht war, daß Erbrechen und Stuhl und Windverhaltung andauerten, aber die Schmerzen sich ausschließlich auf die Bauchgegend und die benachbarten Teile des Unterleibes beschränkten, desgleichen die Druckempfindkeit, und da $B$ keine Spannung des Bauches und nur geringer Meteorismus bestand, d. h. mit anderen Worten, da $B$ nach I4 Stunden die Symptome einer allgemeinen Peritonitis fehlten, die doch wohl nach dieser Zeit bei Entleerung von Darminhalt ins Peritoneum immer vorhanden sind.

Eine Differentialdiagnose zwischen dieser Art von Darmruptur, die sich lediglich im Bauchsack abspielt, und zwischen einer Massenreduktion dagegen wird nur dann möglich sein, wenn in der Bauchhöhle ein zirkumskripter Tumor fühlbar ist. Bei 5 von den 8 
Beobachtungen handelt es sich um Hernien, die schon längere Zeit bestanden und die wiederholt reponiert wurden. Es wäre daher möglich, daß durch die mannigfachen vorausgegangenen Taxisversuche Veränderungen am Darm zustande gekommen sind, die die Elastizität und überhaupt die Konsistenz der Darmwand beeinträchtigen. Bei dem $\mathrm{Schn}$ itzle rschen Fall ist diese Annahme ausgeschlossen, denn Schnitzler erwähnt ausdrücklich, da $\$$ es sich bei seinem Fall um Zerreißung eines bisher offenbar gesunden Darmstücks handelte, wobei man annehmen konnte, da $B$ die Abwesenheit von Bruchwasser, welches sonst nach hydrostatischen Gesetzen den Druck auf die gesamte Darmoberfläche verteilt, in diesem Falle es bewirkt hat, da $B$ der am zentralen Teile der Bauchgeschwulst gepreßte Darm an der periphersten Stelle auseinander riß.

Was die Untersuchung meiner Fälle nach dieser Richtung betrifft, so ist darüber zu sagen, daB bei Fall I, 41/2 Stunden vor der Taxis, die elastische Einklemmung begann und da $B$ in dieser Zeit sehr wohl durch Druckstauung, die zu venöser Hyperämie, Gefäßzerreißungen und konsekutiver Darmlähmung führte, die Widerstandskraft der eingeklemmten Schlinge herabgesetzt sein konnte. Bei Fall 2 bestanden dagegen die Hernie noch nicht lange und wurde durch ein Bruchband so gut zurückgehalten, da $B$ sie nicht austrat und niemals Beschwerden machte. Die Einklemmung bestand vor der Taxis nur $2 \frac{1}{2}$ Stunden und wurde angeblich sehr schonend vorgenommen. Bei der Operation fanden sich an der perforierten Darmschlinge mit Ausnahme der Perforation keine krankhaften Veränderungen, und bei der Naht zeigte die Darmwand normale feste Konsistenz. Die Einklemmung war überdies keine sehr enge. Auch hier ist kein Grund vorhanden, eine Erkrankung des Darms, die eine besondere Brüchigkeit seiner Wandungen zur Folge gehabt hätte, anzunehmen. $G$ a $r \mathrm{r}$ è hat eine Darmperforation, bedingt durch Abreißen eines Appendix epiploicus mit Kotaustritt in den Bruchsack bei sonst gesundem, keineswegs brüchigem Darm gesehen.

Es ist daher nicht daran zu zweifeln, da $B$ auch bei kurzer Dauer der Inkarzeration und bei durch die Inkarzeration wenig geschädigtem Darm eine Ruptur durch Taxis möglich ist.

Die Prognose der sich selbst überlassenen Darmruptur durch 
die Taxis ist eine sehr schlechte. Um so erfreulicher ist es, daß die Resultate bei frühzeitiger Intervention recht gute sind. Bei der Kranken, wo sich die Ruptur in dem eng abgeschnürten Bruchsack abspielte, gelangte eine I4 Stunden nach erfolgter Perforation vorgenommene, größere Darmresektion anstandslos zur Heilung. Von den übrigen Kranken mit Peritonitis, die 4, I8 und 20 Stunden nach der Taxis operiert wurden, kamen sämtliche durch; in einem Fall ist über den Ausgang nichts erwähnt, 2 Kranke wurden nicht operiert und starben und einer starb trotz der 24 Stunden nach der Taxis vorgenommenen Operation.

Erwähnenswert ist noch, daß die Auffindung der Perforationsstelle in keinem Fall erhebliche Schwierigkeiten machte.

\section{Literaturverzeichnis.}

I) Linhardt, Vorlesungen über Unterleibshernien. Würzburg I866.

2) Maydl, Die Lehre von den Unterleibsbrüchen.

3) Schmidts Jahrbücher i $86_{3}$.

4) Frank, R., Perforationsperitonitis durch Ruptur des Darmes bei der Taxis.

5) Krafft, B., Úber lokale und allgemeine Schädigungen infolge von Taxisversuchen inkarzerierter Hernien. Beiträge zur klinischen Chirurgie, 3I. Bd., 2. Heft.

6) Schnitzler, F., Über Hernienruptur durch Selbsttaxis. Wiener klin. Wochenschrift I 899 , Nr. I.

7) Garrè, Die Taxis bei eingeklemmten Hernien. Münchener med. Wochenschr. 1897, Nr. 22. 


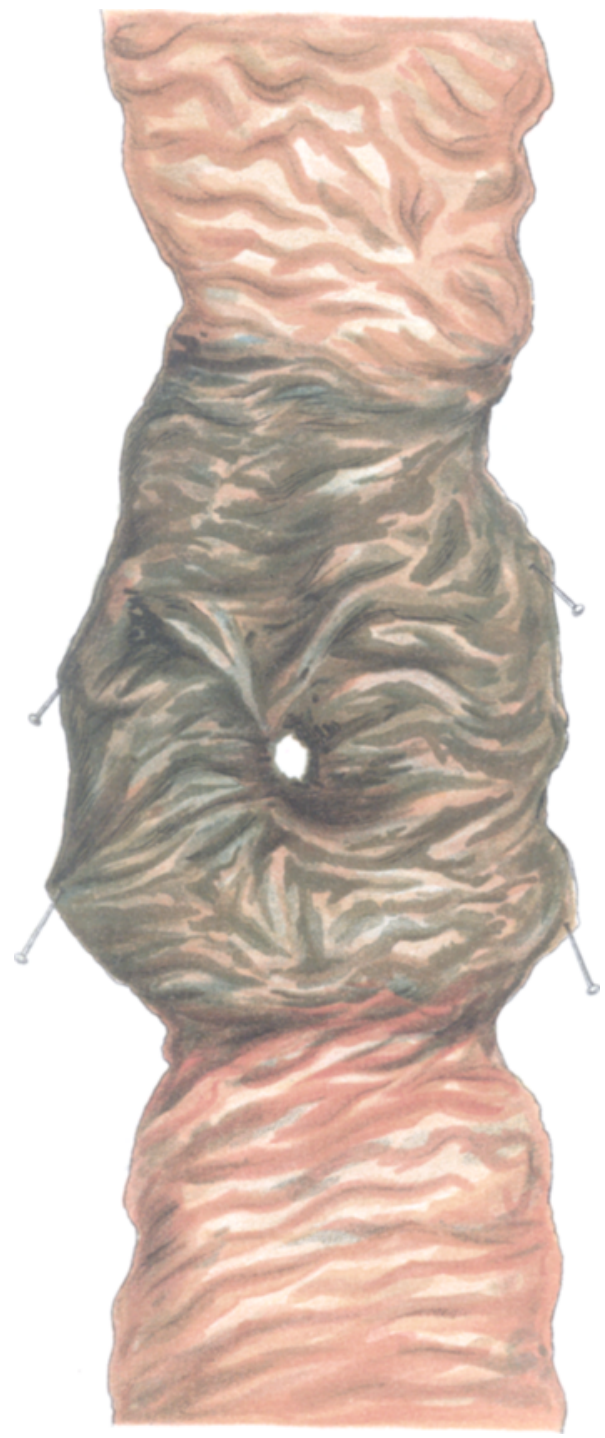

